\title{
Effect of body weight on static and dynamic posturography
}

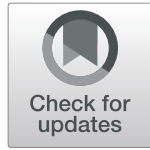

\author{
Afaf Emara', Salwa Mahmoud ${ }^{2 *}$ and Mohamed Emira ${ }^{3}$
}

\begin{abstract}
Background: Postural balance is important to allow us to maintain the daily life. Poor postural response and reduced motor activity were shown to be most prominent among obese persons than in non-obese. It is found that obese elderly groups show a high prevalence of fall and supposed that adipose tissue distribution may be considered as a major factor to balance problems. In this research, we studied computerized dynamic posturography (CDP) static and dynamic tests among healthy adults according to their BMl classification (underweight/normal weight/ overweight/obese).

Results: We found a significant difference between studied groups among the different scores recorded from sensory organization test and rhythmic weight shift test, which means the presence of an effect of increased body mass index on the different sensory systems required to maintain balance control and the motor strategy used to maintain balance. In obese elderly subjects, there was increased reliance on vestibular system to maintaining balance. With increasing BMl, there was decrease in visual dependence in maintaining balance.

Conclusions: Increased body weight affects the balance function of the normal individual. Computerized dynamic posturography is a good test to study static and dynamic status among obese subjects. So we found a positive relation between aging, BMl, and postural control.
\end{abstract}

Keywords: Body weight, Posturography, Sensory organization, Rhythmic weight shift

\section{Background}

Postural balance is needed to allow us to maintain the normal daily life. Balance is considered as the ability to maintain the center of mass $(\mathrm{COM})$ within the base of support (BOS) that will assist to maintain body equilibrium [1].

Postural control is a complex system that helps person to maintain balance in quiet standing position. The information needed in order to maintain balance is monitored by vestibular, proprioception, and visual systems [2].

Computerized dynamic posturography (CDP) is a noninvasive method used to assess the central nervous system adaptive methods (sensory, motor, and central) needed in the control of posture and balance, both in normal and abnormal conditions particularly in the

\footnotetext{
* Correspondence: salwa672000@yahoo.com; salwa672000@yahoo.com

${ }^{2}$ Audiology Department, Hearing \& Speech Institute, Giza, Egypt

Full list of author information is available at the end of the article
}

diagnosis of balance problems needed in posture and balance. CDP requires specific protocols needed to diagnose any affection of the patient's posture control system. Thus, CDP challenges it by using specific combinations of visual and support surface stimuli. The test protocols are the sensory organization test (SOT), limits of stability test (LOS), the motor control test (MCT), and the adaptation test (ADT) [3].

Postural affection and decreased motor activity were shown to be the most obvious among obese children than in non-obese children [4].

Fjeldstad et al. [5] found that obese elderly persons show a high ability to fall and assumed that adipose tissue distribution may be considered as a major cause in balance problems. Increased obesity can affect postural control which is reflected on postural sway and motor reaction time [6]. 
Body mass index (BMI) can be calculated from a person's weight and height [7]. BMI can be measured as body weight divided by the square of body height in meter. The World Health Organization categorizes BMI into four degrees: underweight $\left(<18.50 \mathrm{~kg} / \mathrm{m}^{2}\right)$, normal weight $(18.50$ $\left.24.99 \mathrm{~kg} / \mathrm{m}^{2}\right)$, overweight $\left(25.00-29.99 \mathrm{~kg} / \mathrm{m}^{2}\right)$, and obese $\left(>30 \mathrm{~kg} / \mathrm{m}^{2}\right)[2]$.

\section{Aim of the work}

Study (CDP) static and dynamic tests among healthy adults according to their BMI classification (underweight/ normal weight/overweight/obese).

\section{Methods}

Subjects

I. Control group: consisted of 15 adult persons with normal body weight with their BMI between 18.5 and $24.99 \mathrm{~kg} / \mathrm{m}^{2}$.

II. Study group: was classified according to body mass index (BMI) into three subgroups:

Subgroup (1) (underweight): consisted of 15 subjects with their BMI $<18.5 \mathrm{~kg} / \mathrm{m}^{2}$

Subgroup (2) (overweight): consisted of 15 subjects with their BMI between 25 and $29.99 \mathrm{~kg} / \mathrm{m}^{2}$

Subgroup (3) (obese): consisted of 15 subjects with their BMI $>30 \mathrm{~kg} / \mathrm{m}^{2}$

The study included adult persons with normal hearing aged 18-60.

Exclusion criteria: Patients who have history of:

- Lower limb injury

- Imbalance disorders.

- Vestibular impairment.

- Neurological affection

- Hearing loss

- History of ototoxic or vestibulotoxic treatment

\section{Ethical aspects}

A written consent was signed by all subjects showing their acceptance regarding participation in this study. Each subject was informed about all steps and any possible side effects.

\section{Equipment}

Computerized dynamic posturography, Neurocom version 4 Smart balance Master.

\section{Procedure}

All subjects were submitted to the following:

1. Full history taking

2. Otological examination
3. Body mass index measurement using the formula:

BMI $=$ Body mass $/$ height $^{2}$

4. Posturography testing:

i. Sensory organization test (SOT)

The test assesses equilibrium score, sensory analysis, strategy analysis, and center of gravity in 6 different sensorial conditions:

Condition 1: Fixed surface and visual surround, eyes open

Condition 2: Fixed surface, eyes closed

Condition 3: Fixed surface, eyes open, moving surrounding

Condition 4: Moving surface, eyes open, fixed surrounding

Condition 5: Moving surface, eyes closed

Condition 6: Moving surface, eyes open, moving surrounding

\section{ii. Motor control tests}

Rhythmic weight shift: It assesses the voluntary ability to move the COG from right to left and forward to backward between two targets at slow (3 s peak to peak), medium ( $2 \mathrm{~s}$ peak to peak), and fast (1 s peak to peak) [8].

Table 1 Comparison of equilibrium score of sensory organization test between studied groups

\begin{tabular}{lllllll}
\hline & NW & UW & OW & OB & $P$ value & Sig. \\
\hline SOT1 & 95.5 & 95.1 & 94.6 & 94.5 & 0.49 & NS \\
SD & $(2.03)$ & $(1.7)$ & $(2.1)$ & $(1.6)$ & & \\
SOT2 & 92.9 & 94 & 92.1 & 91.9 & $0.05^{*}$ & S* $^{*}$ \\
SD & $(2.3)$ & $(1.5)$ & $(2.5)$ & $(2.5)$ & & \\
SOT3 & 92.2 & 91.3 & 90.9 & 89.7 & 0.14 & NS \\
SD & $(2.3)$ & $(3.2)$ & $(2.7)$ & $(3.2)$ & & \\
SOT4 & 85.1 & 87.6 & 87.1 & 78.9 & $0.00^{*}$ & S** \\
SD & $(7.1)$ & $(5.2)$ & $(5.9)$ & $(7.5)$ & & \\
SOT5 & 66.5 & 71.3 & 64.3 & 60.5 & 0.15 & NS \\
SD & $(8.7)$ & $(13.8)$ & $(15.4)$ & $(12)$ & & \\
SOT6 & 60 & 64.4 & 61.1 & 56.9 & 0.70 & NS \\
SD & $(15.3)$ & $(21.3)$ & $(18.1)$ & $13.7)$ & & \\
Composite & 73.8 & 74.3 & 72.9 & 70.8 & 0.65 & NS \\
SD & $(8.1)$ & $(6.9)$ & $8.6)$ & $(7.9)$ & & \\
\hline SOT, sensory & or & (6). & & & \\
\hline
\end{tabular}

SOT, sensory organization test equilibrium from condition 1 to 6 , Composite, sensory organization test equilibrium score composite score; NW, normal weight; UW, underweight; $O W$, overweight; $O B$, obese. $S^{*}$, significant difference between UW and $O W$ and between $U W$ and $O B ; S^{* *}$, significant difference between NW and $\mathrm{OB}$, between group $\mathrm{UW}$, and $\mathrm{OB}$ and between $\mathrm{OW}$ and $\mathrm{OB}$ *The mean difference is statistically significant at $P \leq 0.05$ level 


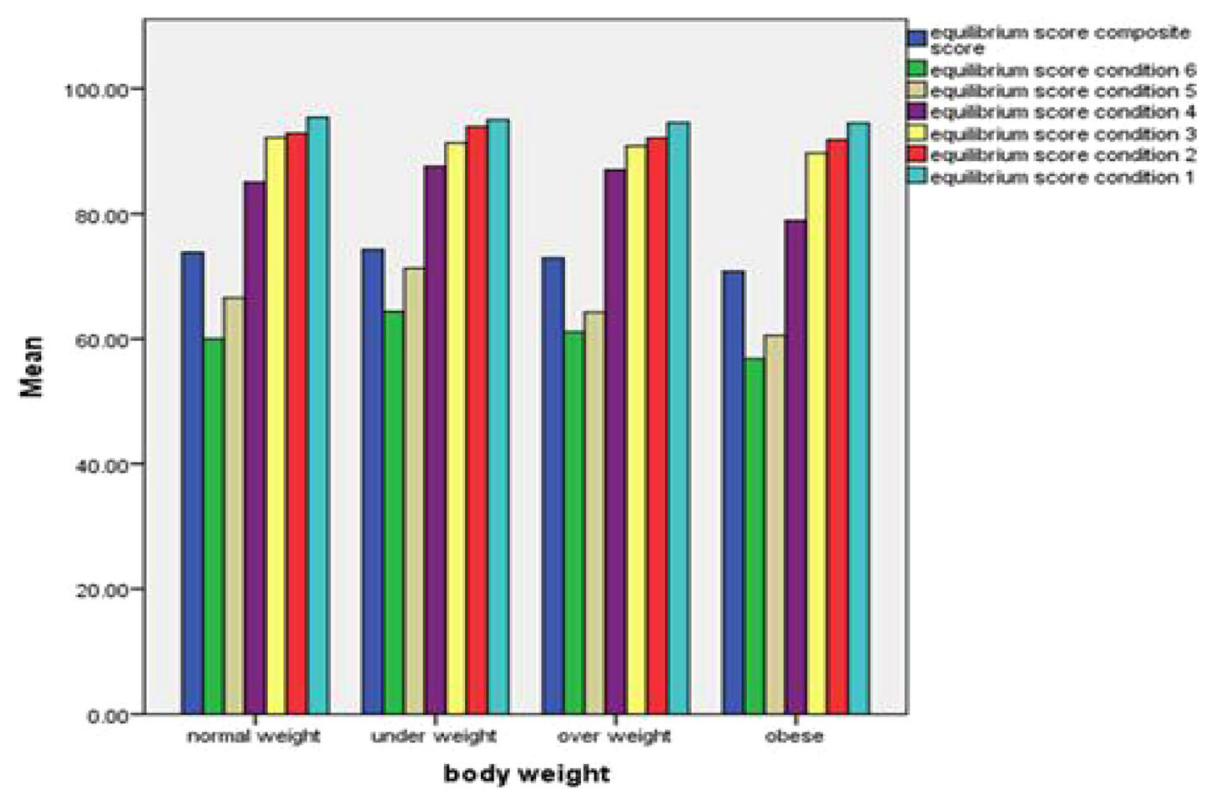

Fig. 1 Comparison of equilibrium score of sensory organization test between studied groups

Movement velocities of the target were $2.67^{\circ} / \mathrm{s}$ (slowmediolateral), $4 \% \mathrm{~s}$ (medium-mediolateral), $8 \%$ (fastmediolateral), $1.78^{\circ} / \mathrm{s}$ (slow-anterior posterior), $2.68^{\circ} / \mathrm{s}$ (medium anterior-posterior), and $5.35 \%$ s (fast anteriorposterior).

1) Directional control: This is a ratio of the amount of movement in the intended direction to the amount of deviation from the ideal movement.

2) On axis velocity: This is the speed of COG movement in the intended direction.

Statistical analysis of the amplitude and latency data was carried out using SPSS system (Statistical Package for Social Sciences) (version16, IBM Corporation ,USA).

\section{Results}

This study included 60 subjects, 44 males and 16 females. Their age range was 20 to 60 years with mean and SD of $30.5 \pm(8.67)$. They were divided into 4 groups:

Control group: Consisted of 15 subjects; body mass index range was 19 to $24 \mathrm{~kg} / \mathrm{m}^{2}$ with mean and SD of $21.7 \pm(1.57)$.

Underweight group: Consisted of 15 subjects; body mass index range was 15 to $17 \mathrm{~kg} / \mathrm{m}^{2}$ with mean and SD of $16.2 \pm(0.77)$.

Overweight group: Consisted of 15 subjects; body mass index range was 26 to $30 \mathrm{~kg} / \mathrm{m}^{2}$ with mean and SD of $27.3 \pm(1.33)$.
Obese group: Consisted of 15 subjects; body mass index range was 31 to $35 \mathrm{~kg} / \mathrm{m}^{2}$ with mean and SD of 33 $\pm(1.41)$.

\section{Sensory organization test}

There was a statistically significant difference between groups in conditions 2 and 4 . There is a high score of underweight subjects in contrast to overweight and obese subjects in sensory organization test equilibrium score in condition 2. In condition 4, we found better score in normal and underweight subjects, as compared to obese subjects and overweight (Table 1 and Fig. 1).

Table 2 Comparison of strategy analysis scores of sensory organization test between studied groups

\begin{tabular}{lllllll}
\hline & NW & UW & OW & OB & $P$ value & S \\
\hline Condition 1 & 99.7 & 99.3 & 99.9 & 90.6 & 0.42 & NS \\
SD & $(1.03)$ & $(1.4)$ & $(0.5)$ & $(1.01)$ & & \\
Condition 2 & 99.2 & 99.8 & 99.5 & 99.2 & 0.73 & NS \\
SD & $(2.3)$ & $(0.6)$ & $(1.8)$ & $(1.7)$ & & \\
Condition 3 & 98.8 & 99.5 & 99.1 & 97.8 & 0.32 & NS \\
SD & $(3.4)$ & $(0.9)$ & $(2.5)$ & $(2.7)$ & & \\
Condition 4 & 82.2 & 83.1 & 82.9 & 73.6 & $0.00^{*}$ & S $^{*}$ \\
SD & $(4.4)$ & $(7.7)$ & $(5.6)$ & $(8.5)$ & & \\
Condition 5 & 67.3 & 69.9 & 65.5 & 59.5 & 0.12 & NS \\
SD & $(1.02)$ & $(12.9)$ & $(10.1)$ & $(12.4)$ & & \\
Condition 6 & 60.4 & 65.4 & 59.3 & 54.3 & 0.26 & NS \\
SD & $(14.7)$ & $(11.4)$ & $(15.6)$ & $(15.6)$ & & \\
\hline
\end{tabular}

NS no statistically significant difference, $S^{*}$ significant difference between NW and $\mathrm{OB}$, between group UW, and $\mathrm{OB}$ and between $\mathrm{OW}$ and $\mathrm{OB}$ *The mean difference is statistically significant at $P \leq 0.05$ level 


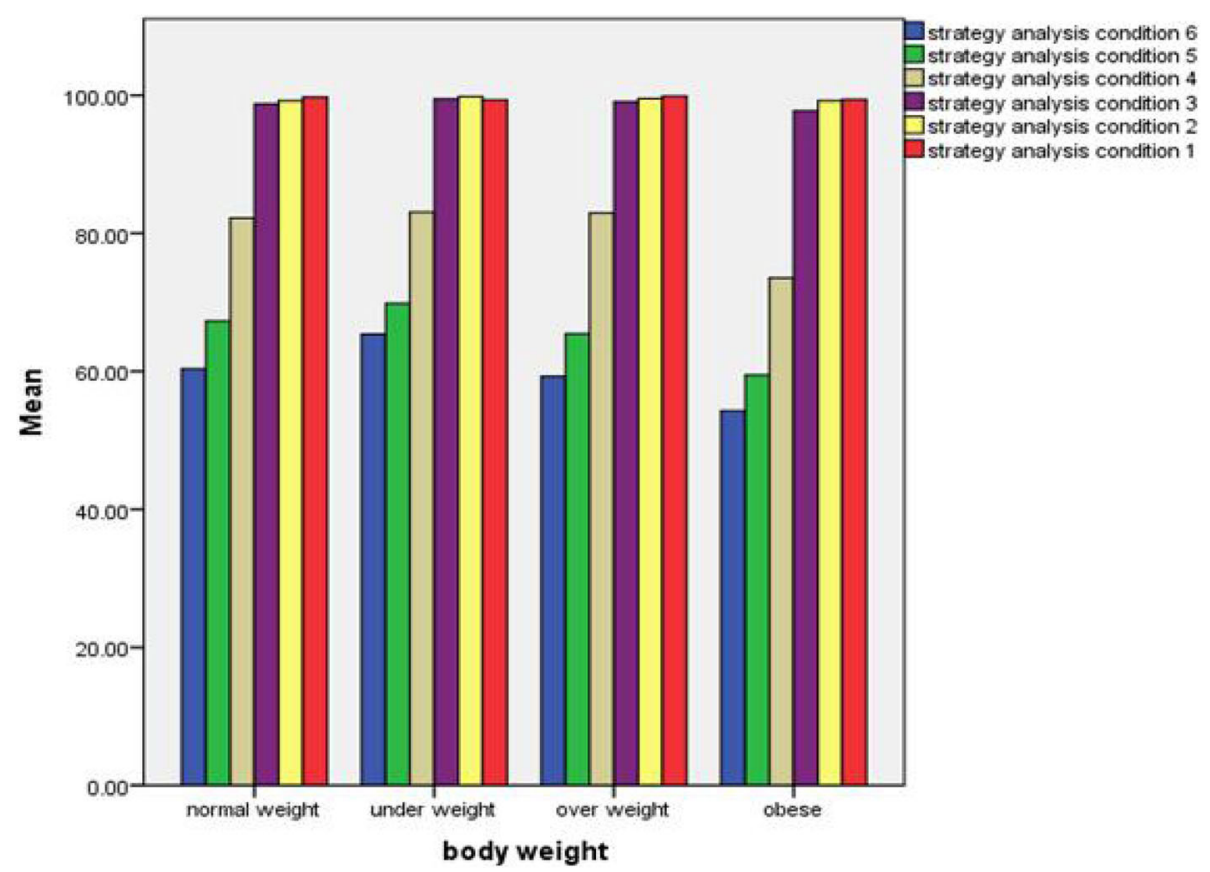

Fig. 2 Comparison of strategy analysis scores of sensory organization test between studied groups

\section{Strategy analysis}

There was a statistically significant difference between groups in condition 4. A better score was found in normal weight and underweight subjects when compared with obese subjects, and better score in overweight when compared with obese subjects in condition 4 scores (Table 2 and Fig. 2).

\section{Sensory analysis}

As regards somatosensory(SOM) and visual (VIS) scores, a statistically significant difference was found between underweight and normal-weight subjects in one side and on the other side overweight and obese subjects (Table 3 ad Fig. 3).

\section{Rhythmic weight shift}

There was a statistically significant difference in fast mediolateral and composite mediolateral scores with better score in normal weight and underweight subjects when compared to obese subjects in rhythmic weight shift (Table 4 and Fig. 4).

When comparing directional control scores of rhythmic weight shift test between studied groups, it was found that there was no statistically significant difference (Table 5 and Fig. 5).

\section{Discussion}

In the present study, we used computerized dynamic posturography, sensory organization test and rhythmic weight shift test, and we determined the effect of body mass index on test results.

As regards the SOT equilibrium score, we detected the presence of significant difference between studied groups in SOT equilibrium score in condition 2 with obese and overweight subjects as they had lower scores than underweight subjects which means that with eyes closed with fixed platform overweight and obese subjects had more anteroposterior sway than underweight subjects.

Table 3 Comparison of sensory analysis scores of sensory organization test between studied groups

\begin{tabular}{lllllll}
\hline & NW & UW & OW & OB & $P$ value & $S$ \\
\hline SOM & 73.8 & 98 & 96.3 & 91.2 & $0.00^{*}$ & $S^{*}$ \\
SD & $(8.1)$ & $(1.6)$ & $(3.2)$ & $(11.1)$ & & \\
VIS & 87.4 & 85.9 & 88.5 & 77.3 & $0.01^{*}$ & $S^{* *}$ \\
SD & $(7.4)$ & $(9.3)$ & $(7.9)$ & $(13.4)$ & & \\
VEST & 63.8 & 64.6 & 59.8 & 63.9 & 0.82 & NS \\
SD & $(10.8)$ & $(16.6)$ & $(15.4)$ & $(17.3)$ & & \\
PREF & 91.9 & 90.3 & 90 & 89.9 & 0.92 & NS \\
SD & $(7.8)$ & $(11.03)$ & $9.1)$ & $7.03)$ & &
\end{tabular}

SOM, somatosensory; VIS, visual; VEST, vestibular; PREF, preference; NS, no statistically significant difference; $S^{*}$, significant difference between NW and UW, between NW and OW, and between NW and OB; $S^{* *}$, significant difference between NW and OB between UW and OB, and between OW and $\mathrm{OB}$

*The mean difference is statistically significant at $P \leq 0.05$ level 


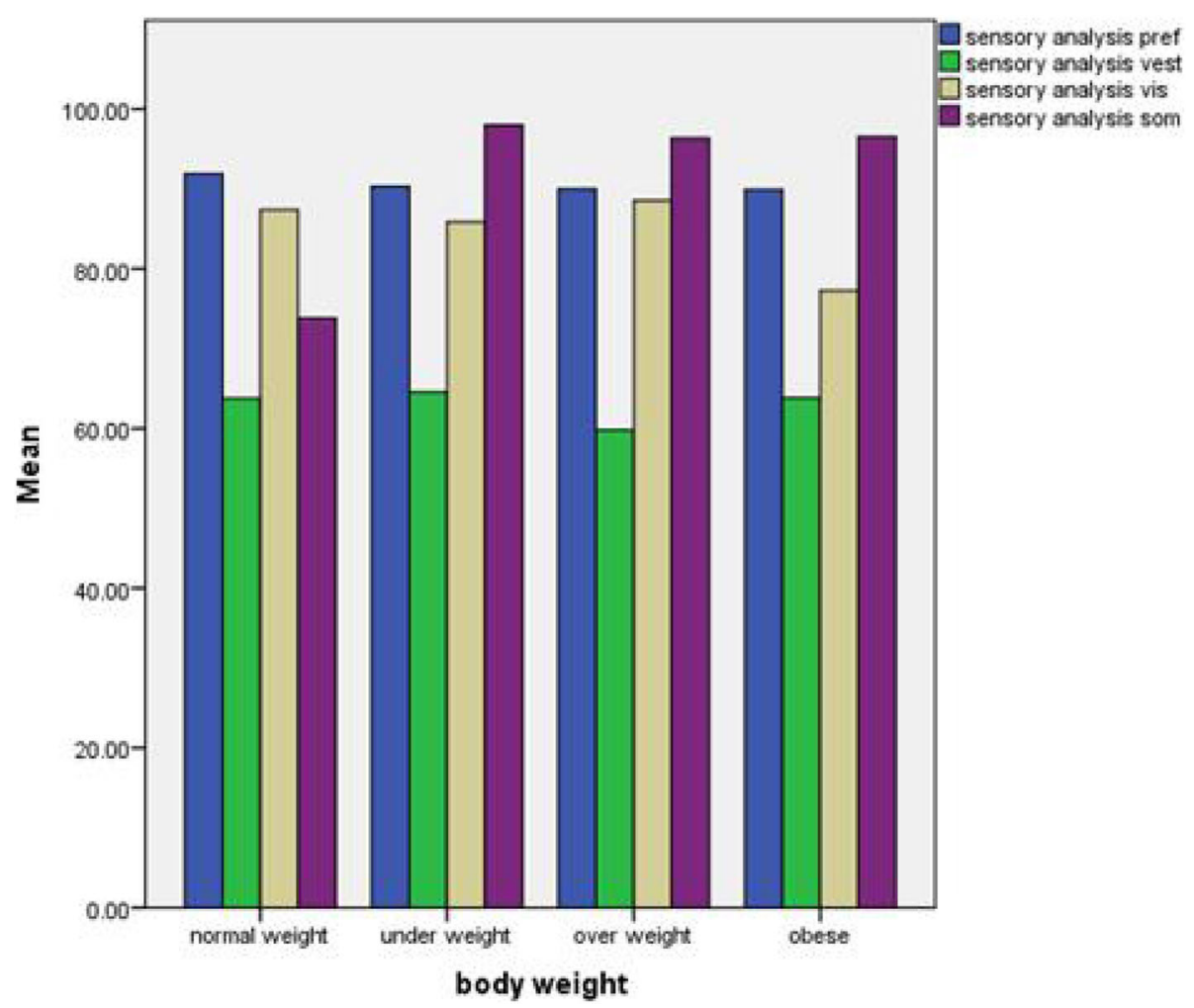

Fig. 3 Comparison of sensory analysis score of sensory organization test between studied groups

Table 4 Comparison of on axis velocity scores of rhythmic weight shift between studied groups

\begin{tabular}{lllllll}
\hline & NW & UW & OW & OB & $P$ value & S \\
\hline SML & 3 & 3.02 & 2.9 & 2.8 & 0.49 & NS \\
SD & $(0.5)$ & $(0.5)$ & $(0.5)$ & $(0.5)$ & & \\
MML & 4.8 & 4.5 & 4.7 & 4.5 & 0.62 & NS \\
SD & $(0.9)$ & $(0.8)$ & $(0.8)$ & $(0.8)$ & & \\
FML & 8.9 & 7.2 & 8.04 & 7.7 & $0.01^{*}$ & $S^{*}$ \\
SD & $(2.1)$ & $(1.8)$ & $(1.9)$ & $(2.1)$ & & \\
CML & 5.6 & 4.9 & 5.2 & 4.7 & $0.04^{*}$ & S** \\
SD & $(1.01)$ & $(0.8)$ & $(0.9)$ & $(0.9)$ & & \\
SAP & 2.1 & 1.9 & 2.3 & 2.2 & 0.77 & NS \\
SD & $(0.4)$ & $(0.5)$ & $(1.03)$ & $1.4)$ & & \\
MAP & 3.2 & 2.9 & 3.1 & 2.8 & 0.65 & NS \\
SD & $(0.7)$ & $(0.5)$ & $(1.02)$ & $(1.1)$ & & \\
FAP & 5.2 & 4.8 & 5.04 & 4 & 0.08 & NS \\
SD & $(1.5)$ & $(0.7)$ & $(1.6)$ & $(1.3)$ & & \\
CAP & 3.5 & 3.3 & 3.5 & 3.01 & 0.42 & NS \\
SD & $(0.8)$ & $(0.5)$ & $(1.1)$ & $(0.9)$ & & \\
\hline The & & & & &
\end{tabular}

The mean difference is statistically significant at $P \leq 0.05$ level $S M L$, slow mediolateral; $M M L$, moderate mediolateral; $F M L$, fast mediolateral; $C M L$, composite mediolateral; $S A P$, slow anteroposterior; $M A P$, moderate anteroposterior; FAP, fast antero-posterior; CAP, composite anteroposterior; NS, no statistically significant difference; $S^{*}$, significant difference between NW and $\mathrm{OB}$ and between UW and $\mathrm{OB} ; S^{* *}$, significant difference between $\mathrm{NW}$ and $\mathrm{OB}$ and between $\mathrm{UW}$ and $\mathrm{OB}$
Our results met with Menegoni et al. [9]. They used a force platform to test 64 healthy subjects. They found that there was increased anteroposterior sway in male groups in eye closed condition which may be related to the greater male body mass index. This can be explained by the fact that there was increased body mass in obese subjects which leads to an increase of torque at ankle level.

The results of the present study agreed with Alonso et al. [10]. They found that subjects with lean mass had more postural control in relation to all of the balance variables with eyes opened and eyes closed using portable force platform while they are testing balance function of 100 subjects.

Our results agreed with Olchowik et al. [11] who found that strategy analysis values decreased with increased BMI which can maintain posture. The ankle muscle activity decreased while the hip muscle activity increased in SOT condition 4 (eyes open, moving platform, stationary surrounding) while there was no significant BMI dependence for SOT condition 1 (eyes closed, stationary platform and visual surrounding) and for SOT condition 2 (eyes closed, stationary platform and visual surrounding).

However, Lavie et al. [12] studied 10 healthy persons with dynamic posturography which was done before and after weight load that was applied to the upper part of 


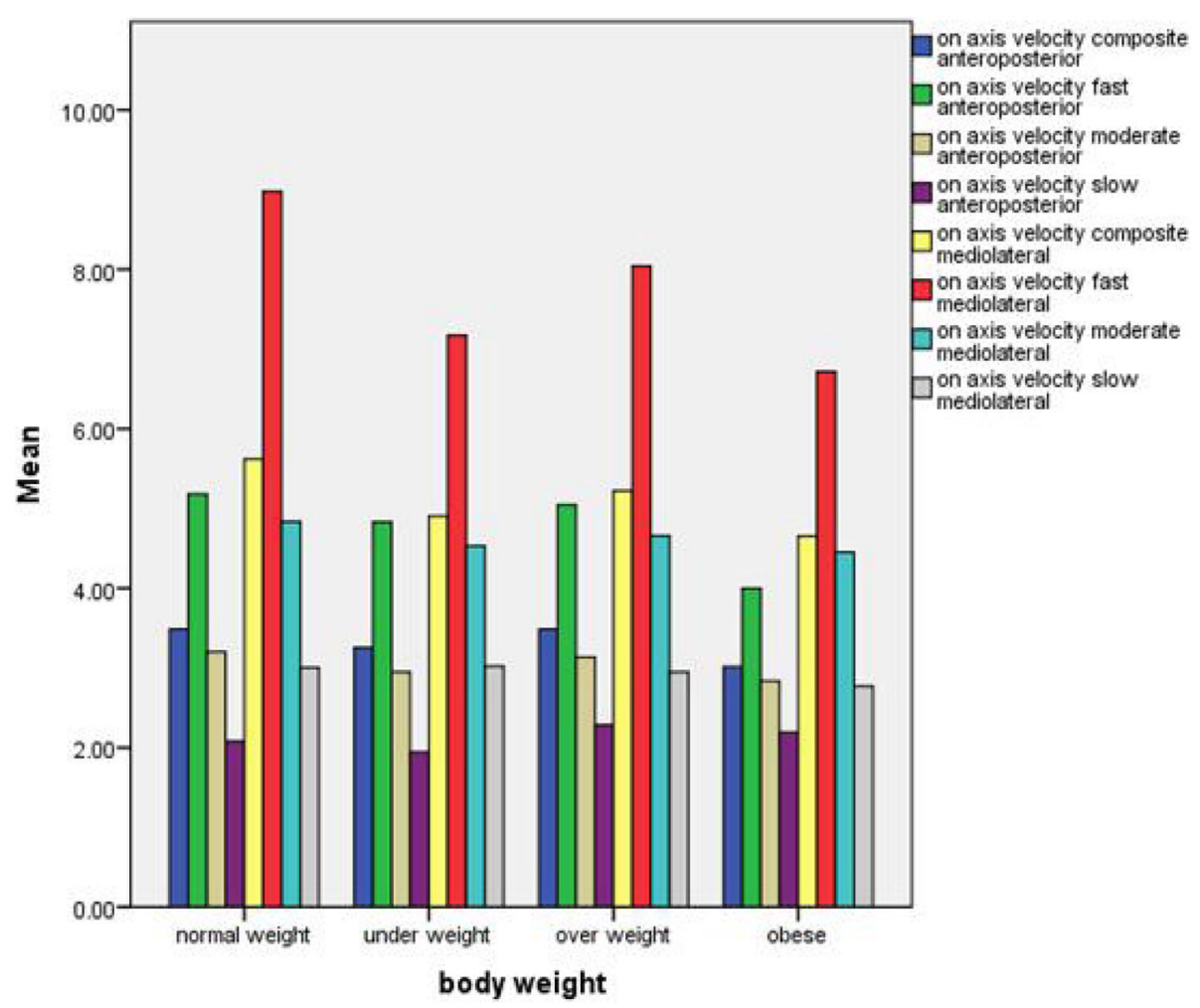

Fig. 4 Comparison of on axis velocity of rhythmic weight shift test between studied groups

Table 5 Comparison of directional control scores of rhythmic weight shift between studied groups

\begin{tabular}{lllllll}
\hline & NW & UW & OW & OB & $P$ value & S \\
\hline SML & 80.4 & 82.7 & 80.5 & 80.3 & 0.59 & NS \\
SD & $(5.9)$ & $(3.2)$ & $(7.3)$ & $(5.5)$ & & \\
MML & 85.1 & 85.1 & 84.5 & 84.2 & 0.93 & NS \\
SD & $(4.5)$ & $(5.2)$ & $(5.4)$ & $(4.1)$ & & \\
FML & 87.5 & 89.1 & 87.1 & 86.1 & 0.23 & NS \\
SD & $(4.1)$ & $(6.4)$ & $(6.4)$ & $(4.9)$ & & \\
CML & 84.3 & 85.9 & 84.1 & 83.5 & 0.44 & NS \\
SD & $(3.5)$ & $(2.9)$ & $(5.7)$ & $(3.7)$ & & \\
SAP & 77.5 & 73.7 & 77.9 & 72.1 & 0.40 & NS \\
SD & $(8.8)$ & $(14.5)$ & $(10)$ & $(10.8)$ & & \\
MAP & 80.3 & 73.9 & 79.3 & 73.2 & 0.41 & NS \\
SD & $(12.1)$ & $(18.2)$ & $(11.1)$ & $(14.5)$ & & \\
FAP & 84.1 & 82.4 & 83.8 & 76.6 & 0.13 & NS \\
SD & $(9.04)$ & $(7.7)$ & $(9.8)$ & $(11.6)$ & & \\
CAP & 80.5 & 76.7 & 80.3 & 73.9 & 0.27 & NS \\
SD & $(9.5)$ & $(12.3)$ & $(8.9)$ & $(10.7)$ & & \\
\hline
\end{tabular}

The mean difference is statistically significant at $\leq 0.05$ level the thorax. They found that with increased weight, only the strategy score difference in (SOT 5) becomes significant, and this was explained that when standing on a movable support surface with absent vision (SOT5) or distorted vision (SOT 6), a tendency was found to use more hip movements to control balance.

Our results agreed with Rossi-Izquierdo et al. [13] who studied balance on a 165 subjects aged 65 years or more. They found that the integration of somatosensory information that related to postural control is not affected by obesity.

Our findings agreed with Matrangola and Madigan [14]; as they studied 135 subjects aged 65 years or more using NeuroCom computerized dynamic posturography, they found that obese people had lower axis velocity scores than non-obese. This agreed with Corbeil et al. [15] who found that a significantly greater ankle torque is needed to stabilize the body in obese individuals, so this fact may interfere their directional control too. They found that directional control scores decrease with increasing BMI.

\section{Conclusions}

1. Increased body weight affects the balance function of the normal individual. 


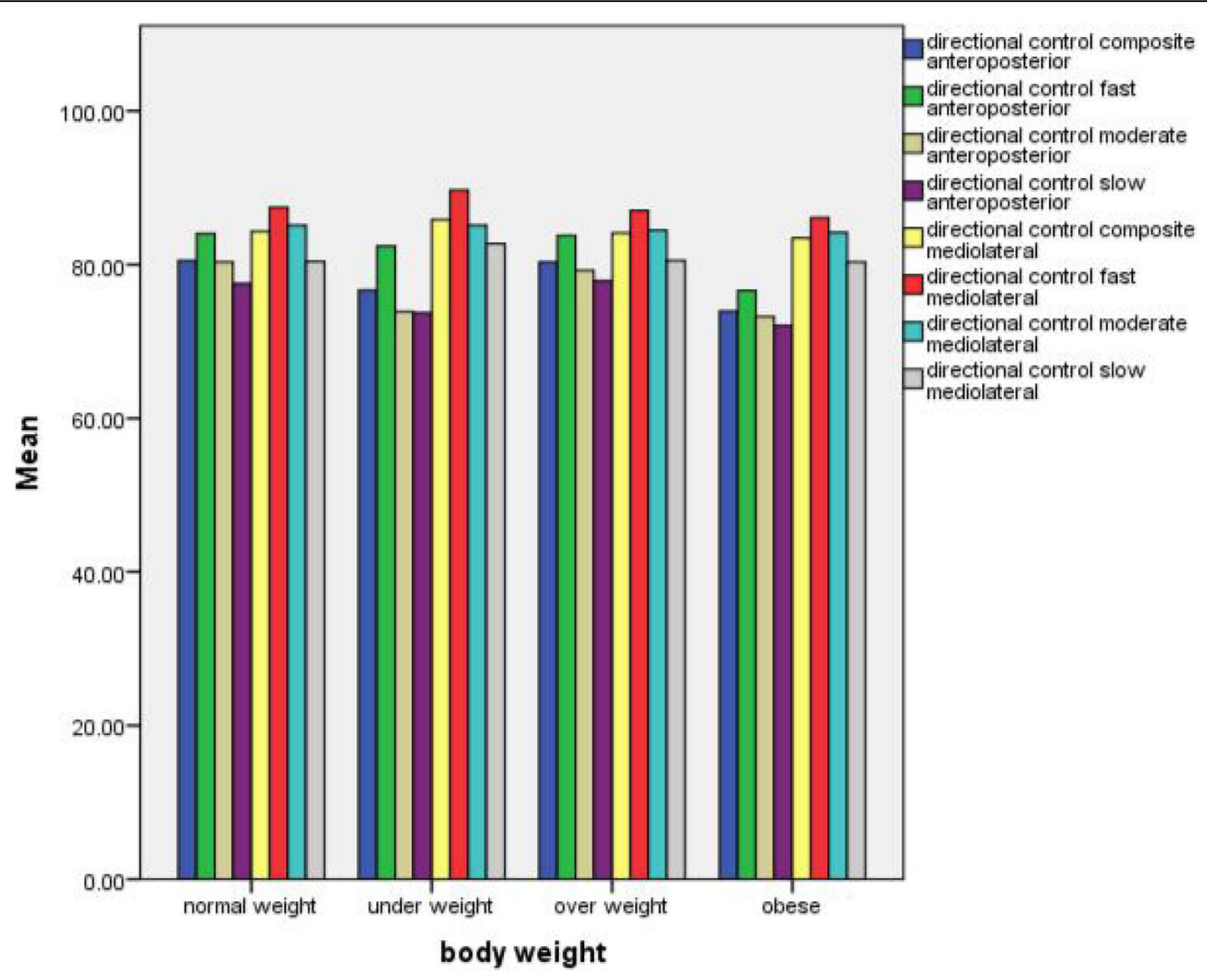

Fig. 5 Comparison of directional control scores of rhythmic weight shift test in studied groups

2. Computerized dynamic posturography is a good test to study static and dynamic status among obese subjects.

\section{Recommendations}

1. The importance of keeping normal weight for better postural control.

2. Including CDP in obese patients with history of imbalance.

3. Obese people should take care during conditions they are exposed with unstable surface or with diminished vision.

\section{Abbreviations}

ADT: Adaptation test; BMI: Body mass index; BOS: Base of support; CDP: Computerized dynamic posturography; COM: Center of mass; LOS: Limits of stability test; MCT: Motor control test; RWS: Rhythmic weight shift test; SOM: Somatosensory; SOT: Sensory organization test; SPSS system: Statistical Package for Social Sciences; VIS: Visual scores

\section{Acknowledgements}

Not applicable

\section{Authors' contributions}

ME: collecting the cases and writing the review; SM: testing with posturography analyzed and interpreted the patient; and AE: statistics and final review of the manuscript. All authors read and approved the final manuscript.

\section{Funding}

None
Availability of data and materials

Information on data supporting the results reported in the article was done in the Audiology Department, Hearing and Speech Institute.

The data used and analyzed during the current study are available from the corresponding author on reasonable request.

Ethics approval and consent to participate

Ethics committee that approved the study: Ethics committee, Faculty of medicine, Tanta University.

The Ethics committee reference number is not available.

A written consent was signed by all subjects showing their acceptance regarding participation in this study. Each subject was informed about all steps and any possible side effects.

\section{Consent for publication}

Not applicable

\section{Competing interests}

The authors declare that they have no competing interests in this section.

\section{Author details}

${ }^{1}$ Audiology Department, Tanta university, Tanta, Egypt. ${ }^{2}$ Audiology Department, Hearing \& Speech Institute, Giza, Egypt. ${ }^{3}$ Audiology Department, Kafr el-Sheikh Hospital, Kafr el-Sheikh, Egypt.

Received: 6 April 2020 Accepted: 20 May 2020

Published online: 19 August 2020

\section{References}

1. Alexandrov A, Frolov A, Horak F, Carlson-Kuhta P, Park S (2005) Feedback equilibrium control during human standing. Biological Cybernetics 93:309-322

2. Karimi N., Ebrahimi I., Kahrizi S. and Torkaman G. (2008): Reliability of postural balance evaluation using the biodex balance system in subjects with and without low back pain " Journal of Postgraduate Medicine 22, 95-10.

3. Ronda JM. (2002): Results of computerized dynamic posturography "spanish otolaryngiology J 53:252-255. 
4. Matter K.C., Sinclair S.A., Hostetler S.G and Xiang H. (2007): A comparison of the characteristics of injuries between obese and non-obese in patients Obesity 15, 2384-2390.

5. Fjeldstad C., Fjeldstad A.S., Acree L.S., Nickel KJ. and Gardner A.W. (2008): The influence of obesity on falls and quality of life" Dynamic Medicine 7, 4-7.

6. Katch V, Becque MD, Marks C, Moorehead C, Rocchini A (1988) Oxygen uptake and energy output during walking of obese male and female adolescents. American J of Clinical Nutrition 47:26-32

7. Meiz Grummer-Strawn LM, Pietrobelli A, Goulding A., Goran Ml and Dietz WH. (2002): Validity of body mass index compared with other bodycomposition screening indexes for the assessment of body fatness in children and adolescents American J of Clinical Nutrition;7597- 985.

8. Boyle JP, Thompson TJ, Gregg EW, Barker LE, Williamson DF (2010) Projection of the year 2050 burden of diabetes in the US adult population: dynamic modeling of incidence, mortality, and prediabetes prevalence. Popul Health Metr 8:29

9. Menegoni F, Galli M, Tacchini E, Vismara L, Cavigioli,Capodaglio P. (2009): Gender-specific effect of obesity on Balance.Obesity 17(10):1951-1956

10. Ange'lica Castilho Alonso,I Nata'lia Mariana S. Luna,ILuis Mochizuki,l Fa'bio Barbieri,ll Sileno Santos,I Julia Maria D’Andre'ía Grevel. (2012) : The influence of anthropometric factors on postural balance: the relationship between body composition and posturographic measurements in young adults, Clinics. 67(12):1433-1441

11. Grazyna Olchowik, Marek Tomaszewsk, Piotr Olejarz,Jan Warchol, Monika Rozanska-Boczula. (2014): The effect of height and BMI on computer dynamic posturography parameters in women, Acta of Bioengineering and Biomechanics Vol. 16, No.

12. Lavie CJ, Milani RV, Ventura HO (2007) Obesity, heart disease, and favorable prognosis-truth or paradox? Am J Med. 120:825-826

13. Marcos Rossi-Izquierdo, Sofi 'a Santos-Pe 'rez ,Ana Faraldo-Garcl', Isabel Vaamonde-Sa 'nchez-Andrade2, Pilar Gayoso-Diz3, Marı 'a Del-Rı 'oValeiras2, Antonio Lirola-Delgado2, Andre 's Soto-Varela2. (2015): Impact of obesity in elderly patients with postural instability, Aging Clin Exp Res 28: 423-428

14. Matrangola SL, Madigan ML (2009) Relative effects of weight loss and strength training on balance recovery. Med Sci Sport Exer 41(7):1488-1493

15. Corbeil P, Simoneau M, Rancourt D, Tremblay A, Teasdale N (2001) Increased risk for falling associated with obesity: mathematical modeling of postural control. IEEE T Neur Sys Reh 9(2):126-136

\section{Publisher's Note}

Springer Nature remains neutral with regard to jurisdictional claims in published maps and institutional affiliations.

\section{Submit your manuscript to a SpringerOpen ${ }^{\circ}$ journal and benefit from:}

- Convenient online submission

- Rigorous peer review

- Open access: articles freely available online

- High visibility within the field

- Retaining the copyright to your article

Submit your next manuscript at $\boldsymbol{\nabla}$ springeropen.com 